\title{
Identification of Ischemic Stroke by Marker Controlled Watershed Segmentation and Fearture Extraction
}

\author{
Mohammed Ajam, Hussein Kanaan, Lina El Khansa, and Mohammad Ayache \\ Department of Biomedical Engineering, Islamic University of Lebanon Beirut, Lebanon
}

\begin{abstract}
In this paper, we will describe a method that distinguishes the ischemic stroke from Computed Tomography (CT) brain images by extracting the statistical and textural features. First, preprocessing of the CT images is done followed by image enhancement. Segmentation of the CT images is performed by Marker Controlled Watershed. After the segmentation, we get the Grey Level Co-occurrence matrix (GLCM) and extract the textural and statistical features. The disadvantage of watershed is the over-segmentation caused by noise and solved by Marker Controlled Watershed as shown experimentally. The features extracted are contrast, correlation, standard deviation, variance, homogeneity, energy and mean. We noticed in our results that the values of homogeneity, energy and mean are bigger in normal CT images than in abnormal CT images where the contrast, correlation, standard deviation and variance of normal CT images are less than those of abnormal CT images (Ischemic Stroke).
\end{abstract}

Keywords: Ischemic Stroke, Watershed, Grey Level Co-occurrence Matrix, Textural and Statistical features.

Received February 27, 2020; accepted June 9, 2020

https://doi.org/10.34028/iajit/17/4A/12

\section{Introduction}

Ischemic stroke is the sudden loss of blood supply to the brain. It is considered as the fifth cause of death in USA [10]. Every year 15 million people suffer from stroke where five million remain untreated and handicapped and five million die [17]. Ischemic stroke cases is 85\% of total stroke cases [11]. In this paper, an algorithm is presented to segment the ischemic stroke Computed Tomography (CT) image and extract the textural and statistical features to identify the stroke easily; especially that $\mathrm{CT}$ scan is available in most hospitals. It requires less scanning time than Magnetic Resonance Imaging (MRI). CT scan is the first imaging modality used to differentiate between Ischemic and Hemorrhagic stroke. In brain CT image, we search for hypo dense changes and White and Grey matter differentiation to suspect a stroke is taking place. We used our algorithm on six patients that consists of three normal Patients and three abnormal patients (having ischemic stroke). The aim is to apply our algorithm to help the non-radiologist identify the stroke both visually and by extracting features that is necessary to next future step, which is the classification of the stroke images.

\section{Literature Review}

Peter et al. [8] compared hemispheres (right and left) of the brain and searched for any differences between changes in ischemia. The regions of stroke detected in the Diffusion Weighted Imaging (DWI) images, and aligned spatially with ncCT images. Image registration was done to do alignment of CT and MR-DW. Yahiaoui and Bessaid [18] used an approach that consists of three stages: preprocessing, image enhancement and classification. First, skull bones and noise are removed, noise are removed by Median filter, and then a multiscale contrast enhancement algorithm based on Laplacian Pyramid (LP). Fuzzy C Means classification applied to extract the ischemic area from normal tissues. Advantage of proposed method is that it shows better results than Discrete Wavelet Decomposition in small size Lesions where it does not show good results in big size lesions and when there is ischemic stroke in both sides (bilateral). Ali et al. [1] segmented the images using Thresholding and Region Growing techniques and extracted the statistical features. Tyan et al. [15] presented a method to detect ischemic stroke detection through an unsupervised feature perception enhancement method consisting of four steps. In the first step, preprocessing is done by curve contrast enhancement cubic method. In the second step, the brain tissue image area is extracted. Abnormal regions detected by an unsupervised region growing algorithm to as a segmentation method and Canny edge detection technologies algorithm (URGA) to make the differences more obvious. The brain is centered horizontally and the white matter is split into eight regions. In the third step, a coinciding regional location method to find the locations of a stroke. Finally, the stroke area is marked with red color. 90 CT images from 26 patients. Results show stroke diagnosis sensitivity of $83 \%$ in comparison to $31 \%$ when radiologists use conventional diagnostic images. 
Gupta et al. [3] segmented the Cerebrospinal Fluid (CSF), White Matter (WM), and Gray Matter (GM) by adaptive thresholding and histogram of $\mathrm{CT}$ images. Most of recent researches are focusing on either CT Perfusion, MRI or MRI Diffusion Weighted Imaging (DWI), which are not available in all hospitals especially in the Middle East, where cost is a major factor to be taken into consideration.

\section{Methodology}

We will describe our methodology summarized in Figure 1 and that will consist of four steps:

1. Preprocessing.

2. Image enhancement.

3. Image segmentation.

4. Feature Extraction.

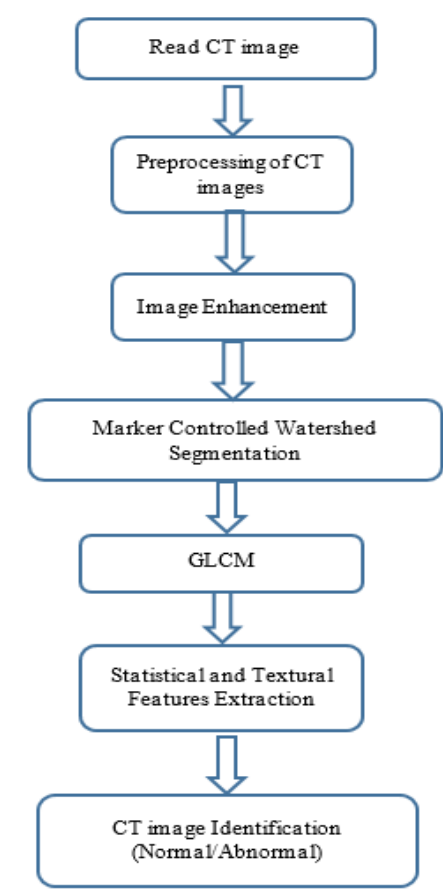

Figure 1. Our algorithm used starting form preprocessing, image enhancement, watershed segmentation, GLCM and feature extraction block diagram.

\subsection{Preprocessing Step}

\subsubsection{Gray Scale Image}

We should change CT image whether it is in Dicom or JPEG format to gray scale. The CT Dicom image is a 16bit image. The CT image contains 4-bit for textural information and 12-bit for display [2]. The CT number (attenuation number) of pixel $(i, j)$ is in Hounsfield Unit (HU) which can be converted to the grey level by Equation (1) [2]:

$$
H U(i, j)=m \times Y(i, j)+a
$$

$Y$ is DICOM grey level, (m) is rescale slope, $a$ is rescale intercept. Those parameters $m$ and $a$ can be found in the textual in-formation in the DICOM image. Usually the dynamic range of $\mathrm{CT}$ image in $\mathrm{HU}$ is from $-1000 \mathrm{HU}$ to
$1000 \mathrm{HU}$. In order to view the image is to convert it into visible 256-greyscale range (lin-ear intensity windowing or window setting). The range of the $\mathrm{HU}$ is called the window width (W) and the center of the range is called the window center $(\mathrm{C})$. The selection of the window width and center is important to review the brain soft tissues. The standard setting is window center of $40 \mathrm{HU}$ and width of $80 \mathrm{HU}$. The window setting is achieved by Equation (2). The HU range outside the window width will be set to level 0 and level 255 .

$$
F(i, j)=(H U(i, j)-(C-W / 2)) / W \times 255
$$

\subsubsection{B-Filtering}

The smoothing Non Linear Filters are many like Min Filter, Max Filter, Mid-Point Filter and Median Filter. We used in our method Median filter as a filtering approach to remove the noises that will cause many problems if not resolved (over segmentation) and affect our results. The Median filter is a non-linear smoothing filter that sorts the intensities of neighborhood pixels of input pixel in ascending order. Then it put the output pixel intensity to be equal to the median of the sorted kernel. It performs better than min, max and mid-point filters by removing many types of noises and less blurring than small sized linear filters [12].

\subsubsection{C-Image Binarization}

The gray image is converted to Binary Image to remove the unneeded data like text (Patient data) and head bone.

\subsubsection{D-Text Removal and Bone Stripping}

A thresholding method is used to remove the skull bone by global thresholding by setting the bone region to zero value and the brain tissue to one value. The average CT intensity is $1000 \mathrm{HU}$ for bones so any threshold less than $200 \mathrm{HU}$ will remove the bone [16]. Morphological operations will be performed to the image so that the eroded binary image is used as a mask for the gray image to get the final skull bone stripped image.

\subsection{Image Enhancement}

Image enhancement is the process that emphasizes, sharpens and feature smoothing for further analysis and processing [16]. Image enhancement can be in the spatial domain where pixels are manipulated or in the frequency domain where spectral components are changed, it can be both the spatial and frequency domains. We used Histogram equalization to enhance our image. Histogram Equalization reset the grey values into new values by a nonlinearly transform function acquired from the normalized cumulative distribution of the image [5]. The grey level $X$ of an image is in the range of $[0,255]$. The Probability Density Function (PDF) of level $X_{k}$ is obtained by Equation (3) [9]:

$$
p\left(X_{-} k\right)=n \_k / n
$$


$n k$ is the number of pixels at level $X_{k} ; \mathrm{n}$ is the total number of pixels in the image. The Cumulative Distribution Function (CDF) of level $X_{k}$ is obtained from the PDF by the Equation (4):

$$
c\left(X_{k}\right)=\sum_{b=0}^{k} p\left(X_{k}\right)
$$

The Transform Function (TF) is given by Equation (5):

$$
T F=\left(X_{-} \max -X_{-} \min \right) C\left(X_{-} k\right)+X_{-} \min
$$

$X \max$ and $X$ min are the maximum and minimum grey level in the output image. The transform function is the normalized CDF when the input image is normalized into grey level of $[0,1]$. The Histogram Equalization stretches the levels with high probability more than the levels with low probability.

\subsection{Segmentation}

We can define Image Segmentation by dividing the image into set of regions. Each region composed of a set of border pixels grouped into forms like a line segments and circular arc segments in images. In addition, it can be defined as group of pixels that has a border and a particular shape like a circle or ellipse or polygon. When the regions of interest do not cover the whole image, image is segmented into foreground regions of interest and background regions that should be ignored [14]. Therefore, aim of image segmentation is discriminating different objects and background in the image. Usually differentiated by [13]:

a) Color, which is the easiest way.

b) Texture, spatial variation in intensity in the image over a certain spatial scale.

c) Motion of an object against a stationary background (by frames).

We used the marker controlled watershed as a segmentation method (based on color and texture variations) summarized in Figure 2.

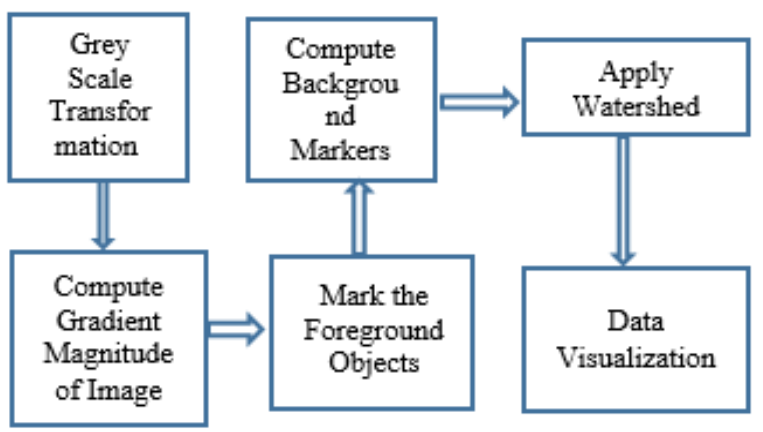

Figure 2. Marker controlled watershed block diagram.

After changing our RGB Image (JPEG or DICOM) into Grey image and doing the preprocessing. Then we get the gradiant image (we used Sobel filter in order to detect all of edges in an image, regardless of direction. Sobel operation has the advantage of providing both a differencing and smoothing effect) [6]. We mark the
Foreground Objects (connected pixels within each object by using morphological operation called "opening-byreconstruction" and "closing-by-reconstruction" creating maxima inside each object, which removes the oversegmentation caused by watershed [8]. After getting the regional maxima of the (opened-closed by reconstruction) image we obtain the foreground markers. In order to mark the background, we get the watershed transform. The watershed transform of the distance transform of binary image allow us to look at the watershed ridgelines of the result. Now, we impose the regional minima and modify the gradient magnitude image so that the regional minima occurs only at foreground and background marker pixels.

\subsection{Feature Extraction}

In order, to extract the features of our CT images we will use the Gray-Level Co-occurrence Matrix (GLCM) which is a statistical method of testing the texture depending on the spatial relationship of pixels by calculating how often pairs of pixel with specific values and in a specified spatial relationship present in an image [7]. We extracted the features energy, correlation, contrast and homogeneity from the GLCM in addition to the mean, standard deviation and variance. In the formulas below $\mathrm{G}$ is maximum grey level and $\mathrm{p}(i, j)$ is the probability for pixel to be at location $(i, j)$ and $\eta$ (mean).

\subsubsection{Energy}

Energy parameter tests the uniformity of the image by returning the sum of squared elements in the GLCM, which is equal to one in constant image and given by the Equation (6) [7].

$$
\sum_{i=0}^{G-1} \sum_{j=0}^{G-1}(p(i, j))^{2}
$$

\subsubsection{Correlation}

Correlation measures how correlated a pixel is to its neighbor over the whole image, which is given by the Equation (7) [7]. $\boldsymbol{\sigma}$ is the standard deviation.

$$
\sum_{i=0}^{G-1} \sum_{j=0}^{G-1} \frac{(i-\mu i)(j-\mu j) p(i, j)}{\sigma i \sigma j}
$$

\subsubsection{Contrast}

Contrast measures the intensity contrast between a pixel and its neighbor over the whole image, which is given by the Equation (8). It provides level of variation in intensities of pixels. It is zero for a constant image [7].

$$
\sum_{i, j}|i-j|^{2} p(h, j)
$$

\subsubsection{Homogeneity}

Homogeneity measures the closeness of the distribution of elements in the GLCM to the GLCM diagonal, which 
is given by the Equation (9) [7].

$$
\sum_{i, j} \frac{(p(i, j))}{1+|i-j|}
$$

\subsubsection{Mean}

Mean gives the contribution of each pixel intensity for entire image, which is given by the Equation (10) [7].

$$
\mu=\sum_{i=0}^{i-1} i p(i)
$$

\subsubsection{Standard Deviation}

Standard deviation shows the variation of set of data around the mean, which is given by the Equation (11) [7].

$$
\sigma=\left(\frac{1}{n-1} \sum_{i=1}^{n}(x i j-\mu j)^{2}\right)^{\frac{1}{2}}
$$

\subsubsection{Variance}

Variance indicates the intensity variations around the mean. It is the average square of the standard deviation, which is given by Equation (12) [7].

$$
\sigma^{2}=\left(\frac{1}{n-1} \sum_{i=1}^{n}(x i j-\mu j)^{2}\right)
$$

\section{Results}

The results obtained from the Marker controlled watershed segmentation and after doing the required processing (filtering and morphological operations), are quiet satisfying as shown in Tables 1, 2, and 3. In Table 1 , the CT images are marked with the markers on the original mage and applying the marker controlled watershed showing good segmentation. In Table 2, we have the CT image of patient 1 who had an ischemic stroke and the infarcted area is on the left side of the brain. We applied the marker-controlled watershed and superimposed the minima markers on the original image. After extending the minima and maxima showing clearly affected area of stroke, especially it is difficult to differentiate between the Gray and White Matter on the right side of the brain tissues. In Table 3, we have CT image of a three normal patients. After applying the Marker Controlled watershed, we superimposed the minima on original image and extended the minima to show the White Matter of the Brain. While extending Maxima that represents the Gray Matter of the brain. Therefore, we are able to differentiate between the Gray and White Matter. In Table 4, we computed the values of textural and statistical features of the six patients CT images. The Energy measures the repeated pairs and is considered high if the number of the repeated pairs is high and gives an indication about the number of greylevel in the image. When its value is low, the homogeneity will be low and means that there is an ischemia. Correlation measures the relevance of grey of pixel whereas the contrast represents the difference between highest and lowest values of a set of pixels.
Therefore, when the contrast value increases the homogeneity decreases, this is also an indication of ischemia. When the homogeneity increases the variance and standard deviation (inform us about the distribution of pixels with respect to the mean) decreases. Our resulting values of contrast, correlation, standard deviation and variance of three normal CT images are less than the values of three abnormal CT images (contains ischemic stroke). The values of the homogeneity, energy and mean are bigger in three normal CT images than those of the three abnormal CT images since abnormal images contain infarcted regions which means less homogeneous therefore, less energy than normal images. Extracting the statistical and textural features values will help to identify the ischemic stroke CT images from the normal ones and important for future classification with increased number of patients. We applied wavelet decomposition using Daubechies (DB) wavelet and obtain the textural and statistical features where the results were not satisfying so we did not include them in this paper. Our proposed method is implemented using Matlab software version 2010.

Table 1. a-CT images of three Ischemic Stroke patients; b- after applying the Marker Controlled Watershed, c-Colored Watershed Matrix (Lrgb).

\begin{tabular}{|c|c|c|c|}
\hline Image Name & a-Original Image & $\begin{array}{c}\text { b-Marker Based } \\
\text { Watershed }\end{array}$ & $\begin{array}{c}\text { c-Colored } \\
\text { Watershed }\end{array}$ \\
\hline Patient 1 & Patient 4 & & \\
\hline Patient 3 & & & \\
\hline
\end{tabular}

Table 2. CT image of Ischemic Stroke patient after applying the Marker Controlled Watershed.

\begin{tabular}{|c|c|}
\hline Skull Stripped Stroke Image & $\begin{array}{c}\text { Minima Marker Superimposed on } \\
\text { Original Image }\end{array}$ \\
\hline Extended Minima & Extended Maxima \\
\hline Watershed & Colored Watershed \\
\hline & \\
\hline
\end{tabular}


Table 3. CT images of three Normal patients showing the extended minima and maxima.

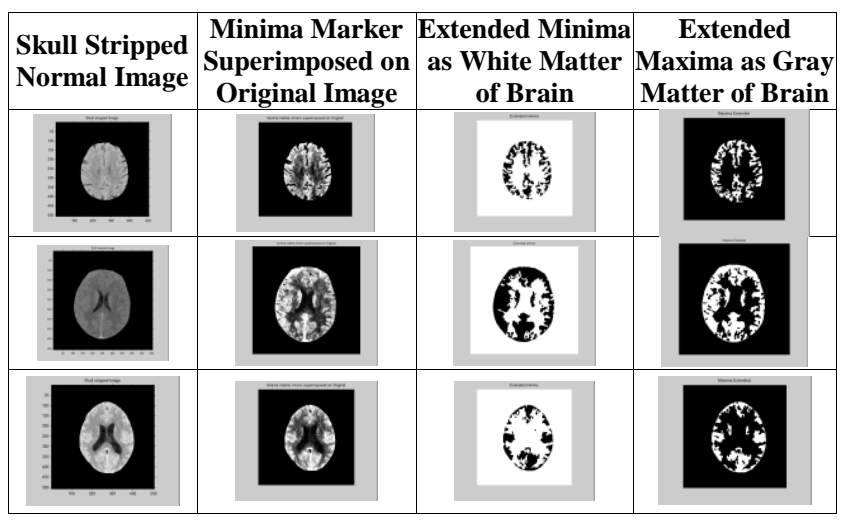

\section{Conclusions}

We conclude that segmentation of brain CT images using Marker watershed showed good results for Ischemic stroke. Where extracting the statistical and textural features are necessary and beneficiary for us, especially it is required for classification as future work with increased number of patients in order to classify and differentiate between Ischemic and normal CT images in addition to developing the graphical user interface. Based on the above results, we can identify the ischemic stroke CT images from the normal CT images by relying on our segmentation method and the features extracted.

Table 4. Textural and Statistical features of the six CT images of patients (Normal and Abnormal).

\begin{tabular}{|c|c|c|c|c|c|c|c|}
\hline Patient CT Image & Contrast & Correlation & Energy & Homogeneity & Mean & Variance & Standard Deviation \\
\hline Image Abnormal 1 & 0.0263 & 0.8883 & 0.7752 & 0.9880 & 250.3613 & 152.1452 & 8.5878 \\
\hline Image Abnormal 2 & 0.0331 & 0.8686 & 0.7656 & 0.9850 & 249.9316 & 184.5612 & 9.1602 \\
\hline Image Abnormal 3 & 0.0457 & 0.8800 & 0.7399 & 0.9840 & 249.0853 & 219.9317 & 10.0352 \\
\hline Image Normal1 & 0.0156 & 0.6911 & 0.9340 & 0.9922 & 253.8026 & 18.5030 & 2.7418 \\
\hline Image Normal2 & 0.0215 & 0.8644 & 0.8312 & 0.9894 & 251.6675 & 89.5484 & 6.4517 \\
\hline Image Normal3 & 0.0172 & 0.8296 & 0.8824 & 0.9914 & 253.2535 & 38.3680 & 4.4183 \\
\hline
\end{tabular}

\section{References}

[1] Ali A., Abdulsalam S., and Nema I., "Detection and Segmentation of Ischemic Stroke Using Textural Analysis on Brain CT Images," International Journal of Scientific and Engineering Research, vol. 6, no. 2, pp. 396-400, 2015.

[2] De Carvalho I., Silva C., De Freitas D., Ambrosano G., Bóscolo F., and Almeida S., "Bone Density: Comparative Evaluation of Hounsfield Units In Multislice And Cone-Beam Computed Tomography," Brazilian Oral Research, vol. 26 no. 6, pp. 550-556, 2012.

[3] Gupta V., Ambrosius W., Qian G., Blazejewska A., Kazmierski R., Urbanik A, and Nowinski W., "Automatic Segmentation of Cerebrospinal Fluid, White and Gray Matter in Unenhanced Computed Tomography Images," Academic Radiology, vol. 17, no. 11, pp. 1350-1358, 2010.

[4] Gonzalez R., Woods R., Eddins S., "Morphological Reconstruction from Digital Image Processing Using Matlab," MatlabDigest,https://ww2.mathworks.cn/tagteam /64199_91822v00_eddins_final.pdf, Last Visited, 2019.

[5] Lucas C., Kemmling A., Mamlouk A., and Heinrich M., "Multi Scale Neural Network for Automatic Segmentation of Ischemic Stroke on Acute Perfusion," in Proceedings of $15^{\text {th }}$ International Symposium on Biomedical Imaging, Washington, pp. 1118-1121, 2018.

[6] Meyer F., "The Watershed Concept and Its Use in Segmentation: A Ariefhistory," https://www.researchgate.net/publication/221661 175_The_watershed_concept_and_its_use_in_se gmentation_a_brief_history, Last Visited, 2019.

[7] Mathworks,https://www.mathworks.com/help/im ages/texture-analysis-using-the-gray-

levelcooccurrence-matrix-glcm.html, Last Visited, 2019.

[8] Peter R., Korfiatis P., Blezek D., Beitia A., Buksakowska I., Horinek D., Flemming K., and Erickson B., "A Quantitative Symmetry-Based Analysis of Hyperacute Ischemic Stroke Lesions in Non-Contrast Computed Tomography," Medical Physics, vol. 44, no. 1, 2017.

[9] Radiopaedia,https://radiopaedia.org/articles/ischa emic-stroke, Last Visited, 2019.

[10] Stroke Association, http://www.strokeassociation.org/STROKEORG/ AboutStroke/Impact-of-Stroke-Strokestatistics_UCM_310728_Article.jsp\#.W1DWGW xNCZ8, Last Visited, 2019.

[11] StrokeAssociation,Internet:http://www.strokeasso ciation.org/STROKEORG/AboutStroke/Impactof-Stroke-Stroke-

statistics_UCM_310728_Article.jsp\#.W1DWGW xNCZ8, Last Visited, 2019.

[12] Shapiro L., Stockman G., "Computer Vision," 2010.

[13] Sharma P., Singh G., and Kaur A., "Different Techniques of Edge Detection in Digital Image Processing," International Journal of Engineering Research and Applications, vol. 3, no. 3, pp. 458461, 2013. 
[14] Shih F., Image Processing and Pattern Recognition: Fundamentals and Techniques, Wiley, 2010.

[15] Tyan Y., Wu M., Chin C., Kuo Y., Lee M., and Chang H., "Ischemic Stroke Detection System with a Computer-Aided Diagnostic Ability Using an Unsupervised Feature Perception Enhancement Method," International Journal of Biomedical Imaging, vol. 2014, pp. 12, 2014.

[16] Umbaugh S., Digital Image Processing and Analysis Human and Computer Vision applications with CVIPtools, CRC Press, 2010.

[17] World Health Organization, Internet: http://www.who.int/topics/cerebrovascular_accid ent/en/, Last Visited, 2019.

[18] Yahiaoui A. and Bessaid A., "Segmentation of Ischemic Stroke Area from CT Brain Images," International Symposium on Signal, Image, Video and Communications, Tunisia, 2016.

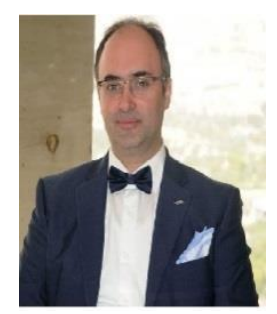

Mohammed Ajam is an experienced 39 years old biomedical engineer born in Tripoli, Lebanon and holds a master degree of biomedical engineering from Islamic University of Lebanon in 2019. Mohammed has three publications mainly in neuro imaging and oncology. Mohammed works as a regional director of Scientech Intervention Company and has an experience more than 10 years in management of sales, marketing, clinical and research of Interventional Neuro and Peripheral medical products in the Middle East.

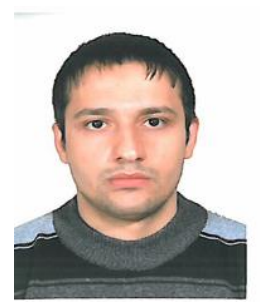

Hussein Kanaan $\mathrm{He}$ received the B.S. degree in biomedical engineering in 2008. In 2012, he received the M.S. degree in communication engineering from Shahed University, Tehran, Iran. He received $\mathrm{Ph} . \mathrm{D}$. degree in electronic engineering from, Shahed University, Tehran, Iran in 2017. Currently, he is an associate professor of Biomedical Engineering Department, Islamic University, Lebanon. His research fields are image processing, signal processing, data mining and machine learning.

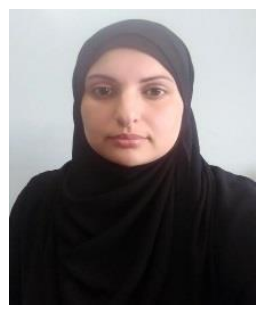

Lina El Khansa obtained a bachelor degree of engineering in biomedical from the Islamic University of Lebanon in 2003. She received the DEA in signals and images from the University of Paris XII, France in 2004. She received the Ph.D. degree in signal processing from the University of Paris EST, France in 2009. She is currently the head of biomedical department at the faculty of engineering at the Islamic university of Lebanon. Her research interests include signal and image processing techniques.

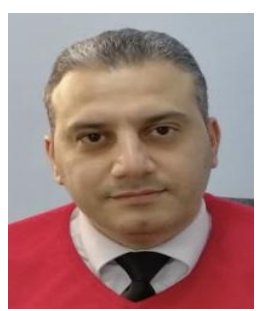

Mohammed Ayache obtained a bachelor degree of engineering in biomedical from the Islamic University of Lebanon. He received the DEA in Signals and Images in biology and medicine from the University of Angers, France in 2004. He received the Ph.D. degree in medical Image Processing from the University of Tours, France, in 2007. He was the head of department of biomedical at the faculty of engineering at the Islamic University of Lebanon from 2009 to 2017 . He was also vice dean of the faculty of engineering from 2014 to 2017. Currently, he is the head of graduate studies at the faculty of engineering. His research interests include advanced neural networks software development and advanced signal and image processing techniques. 